Homology, Homotopy and Applications, vol.20(1), 2018, pp.219-236

\title{
PERFECT DISCRETE MORSE FUNCTIONS ON CONNECTED SUMS
}

\author{
HANIFE VARLI, MEHMETCIK PAMUK AND NEŽA MRAMOR KOSTA
}

(communicated by Donald M. Davis)

\begin{abstract}
We study perfect discrete Morse functions on closed, connected, oriented $n$-dimensional manifolds. We show how to compose such functions on connected sums of manifolds of arbitrary dimensions and how to decompose them on connected sums of closed oriented surfaces.
\end{abstract}

\section{Introduction}

Since it was introduced by Marston Morse in the 1920s, Morse theory has been a powerful tool in the study of smooth manifolds. It allows to describe the topology of a manifold in terms of the cellular decomposition generated by the critical points of a smooth map defined on it. By analyzing the function's critical points, it is possible to construct a cell structure for the manifold.

In the 1990s Robin Forman developed a discrete version of Morse theory that turned out to be an efficient method for the study of the topology of discrete objects, such as regular cell complexes. As in the smooth setting, changes in the topology are deeply related to the presence of critical cells of a discrete Morse function. The analysis of the evolution of the homology of the cell complexes can be a very useful tool, for example in computer vision to deal with shape recognition problems by means of topological shape descriptors, and in topological data analysis, where new information can be extracted from the data. It can also be used for efficient computation of homology of the cell complex $[\mathbf{6}, \mathbf{9}]$. Many of the familiar results from the smooth theory apply in the discrete setting.

A discrete Morse function on a cell complex is an assignment of a real number to each cell in such a way that the natural order given by the dimension of cells is respected, except at most in one (co)face for each cell. A discrete Morse function on a regular cell complex induces a partial pairing on the cells called a discrete vector field. It consists of pairs of cells of two consecutive dimensions on which the function reverses the order given by the dimension. Those cells that do not belong to any pair are precisely the critical cells of the map. A gradient path is a connected sequence of the pairs on which the function is non-increasing. The idea of discrete gradient path plays a central role in Forman's theory, due to the fact that the properties related to the critical elements are more easily visualized. Moreover, the discrete gradient paths

Received December 2, 2016, revised October 5, 2017; published on January 31, 2018. 2010 Mathematics Subject Classification: 57R70, 37E35.

Key words and phrases: perfect discrete Morse function, discrete vector field, connected sum.

Article available at http://dx.doi.org/10.4310/HHA.2018.v20.n1.a13

Copyright (c) 2018, International Press. Permission to copy for private use granted. 
give us an easy and efficient method for getting examples of such functions verifying certain initial conditions (for example, having a given number of critical cells) avoiding rather complicated numerical assignments. On the other hand, since different discrete Morse functions may induce the same discrete gradient field, working with a vector field instead of a Morse function may lead to some disambiguities about values of a function on cells. We will point out this fact again later in Section 3.

The discrete Morse functions that have as few critical cells as possible have been widely studied in the literature (see $[\mathbf{1 0}, \mathbf{1 4}]$ ). The number of critical $n$-cells of a discrete Morse function is greater than or equal to the $n$-th Betti number of the cell complex by Morse inequalities (given in more details on page 3) [6, Corollary 3.7]. For a perfect discrete Morse function these two quantities are the same. Although there are complexes which do not admit perfect discrete Morse functions, these functions are the most suitable for combinatorial and computational purposes and they have been studied greatly.

In this paper we study perfect discrete Morse functions on connected sums of triangulated manifolds and consider the problem of (de)composing such maps as perfect discrete Morse functions on the summands. We show that a perfect discrete Morse function on a connected sum of triangulated manifolds of any dimension is obtained by combining perfect discrete Morse functions on the summands, after possibly minor subdivisons in a neighborhood of the connecting sphere (cf. Theorem 3.1). On decomposing a given perfect discrete Morse function, we consider only the 2-dimensional case (cf. Theorem 4.6). For a triangulated connected sum $M_{1} \# M_{2}$ of two closed connected surfaces with a given perfect discrete Morse function we give an explicit construction of a separating circle $C$ such that the given perfect discrete vector field restricts to a perfect discrete vector field on each summand (cf. Theorem 4.4). Such a construction can be implemented in the form of an algorithm which could be useful in applications of topological methods for example to data, since it enables a subdivision of the data analyzed into smaller, simpler parts.

As we will show in a further paper [13], our proof generalizes to the 3-dimensional case, but in higher dimensions new phenomena appear. One cannot extend the methods that we use in dimensions 2 and 3 to higher dimensional manifolds since in higher dimensions homology groups and the Euler characteristic do not suffice to classify the boundary manifold as a sphere (see Remark 4.7). Moreover, in dimensions greater than 3 a connected sum decomposition of a manifold is not unique.

The paper is organized as follows: In section 2, we recall necessary basic notions of discrete Morse theory for our proof. In section 3, we prove how to compose a perfect discrete Morse function on a connected sum of triangulated $n$-dimensional manifolds. In section 4, we show how to decompose a perfect discrete Morse function on surfaces and give an example about decomposing a perfect discrete Morse function on genus 2 surface.

\section{Acknowledgments}

This research was supported by the Slovenian-Turkish grants BI-TR/12-14-001 and 111T667, and by the ESF Research Network Applied and Computational Algebraic Topology (ACAT). This is a part of the first author's PhD thesis. The authors would 
like to thank the referee for the valuable comments which helped to improve the manuscript.

\section{Preliminaries}

In this section we recall necessary basic notions of discrete Morse theory. For more details we refer the reader to [6] and [7]. Throughout this section $K$ denotes a finite regular cell complex. We write $\tau>\sigma$ if $\sigma \subset \bar{\tau}$ (closure of $\tau$ ).

A discrete Morse function $f: K \rightarrow \mathbb{R}$ on $K$ associates values to the cells of $K$ such that for any $p$-cell $\sigma \in K$ each $(p-1)$-face $\nu<\sigma$ except at most one has value $f(\nu)<f(\sigma)$, and each $(p+1)$-coface $\tau>\sigma$ except at most one has value $f(\tau)>f(\sigma)$. The cells of $K$ are subdivided into critical and regular cells, where the critical cells are cells where none of the above exceptions occur, and regular cells appear in disjoint pairs which form the gradient vector field

$$
V=\{(\sigma, \tau) \mid \operatorname{dim} \sigma=\operatorname{dim} \tau-1, \sigma<\tau, f(\sigma) \geqslant f(\tau)\} .
$$

We draw arrows to represent the vector field as follows: If $\tau^{(p+1)}>\sigma^{(p)}$ and $f(\sigma) \geqslant$ $f(\tau)$ then we draw an arrow from $\sigma$ to $\tau$ as in Figure 1.

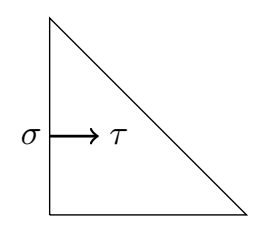

Figure 1: $f(\sigma) \geqslant f(\tau)$.

A cell is critical if and only if it is neither the tail nor the head of an arrow.

Critical cells are related with the topology of the complex as in the case of a smooth manifold with a smooth Morse function, that is, a regular cell complex $K$ with a discrete Morse function $f$ has the homotopy type of a CW complex with cells that correspond to the critical cells of the function, and the number of critical cells is bounded by the Betti numbers of $K$ in terms of Morse inequalities:

1. $m_{p}(f)-m_{p-1}(f)+\cdots \pm m_{0} \geqslant b_{p}-b_{p-1}+\cdots \pm b_{0}$,

2. $m_{p}(f) \geqslant b_{p}$,

3. $m_{0}(f)-m_{1}(f)+\cdots+(-1)^{n} m_{n}(f)=b_{0}-b_{1}+\cdots+(-1)^{n} b_{n}$,

where $b_{p}$ is the $p$-th Betti number of $K$ and $m_{p}(f)$ is the number of critical $p$-cells of $f$ for $p=0,1, \ldots, n$ where $n$ is the dimension of $K$.

Pairs of regular cells connect into gradient paths along which function values of $f$ descend. A gradient path or a $V$-path of dimension $(p+1)$ is a sequence of cells

$$
\sigma_{0}^{(p)} \rightarrow \tau_{0}^{(p+1)}>\sigma_{1}^{(p)} \rightarrow \tau_{1}^{(p+1)}>\cdots \rightarrow \tau_{r}^{(p+1)}>\sigma_{r+1}^{(p)},
$$

such that $\left(\sigma_{i}^{(p)}<\tau_{i}^{(p+1)}\right) \in V$ and $\sigma_{i}^{(p)} \neq \sigma_{i+1}^{(p)}<\tau_{i}^{(p+1)}$ for each $i=0,1, \ldots, r$.

Gradient paths are represented by a sequence of arrows. Since function values descend along a gradient path, gradient paths do not form cycles. In fact, a discrete 
vector field, that is, a collection of disjoint pairs of cells $\left(\tau^{(p+1)}>\sigma^{(p)}\right)$, is the gradient vector field of a discrete Morse function if and only if paths in the vector field do not form cycles [7, Theorem 3.5].

In discrete Morse theory, gradient vector fields are often more useful than the underlying discrete Morse functions for the combinatorial purposes. Let us define an equivalence relation between discrete Morse functions.

Definition 2.1. Two discrete Morse function $f$ and $g$ are called equivalent if for every pair of cells $\left(\tau^{(p)}<\sigma^{(p+1)}\right)$ in $K$,

$$
f(\sigma)<f(\tau) \text { if and only if } g(\sigma)<g(\tau) .
$$

The following theorem gives us ground to work with the gradient vector fields instead of the function values.

Theorem 2.2. ([2]) Two discrete Morse functions $f$ and $g$ defined on a simplicial complex $K$ are equivalent if and only if $f$ and $g$ have the same critical cells and induce the same gradient vector field.

In this paper we study a special type of discrete Morse functions, where the Morse inequalities are actually equalities. As in the smooth case, such Morse functions are called perfect (with respect to the given coefficient ring, which is fixed throughout most of this paper and suppressed in our notation).

Definition 2.3. ([1]) A discrete Morse function $f: K \rightarrow \mathbb{R}$ and its discrete gradient field are called perfect if for each $p$,

$$
m_{p}(f)=b_{p}=\operatorname{rank} H_{p}(K) .
$$

Perfect discrete Morse functions have the least number of critical cells by means of the Morse inequalities. Therefore, they are the most suitable functions for combinatorial and computational purposes. Existence of such functions has both theoretical and practical applications. An arbitrary CW-complex may not have a perfect discrete Morse function defined on it. For example, any torsion in homology is an obstruction to a $\mathbb{Z}$-perfect discrete Morse function and any complex that is acyclic (homologically trivial) and non-collapsible e.g. the dunce hat and Bing's House cannot admit a perfect discrete Morse function see [1, section 4] and [3, page 16]. Also, every sphere of dimension $d>4$ has a triangulation which does not admit a perfect discrete Morse function [5]. On the other hand, it is easy to see that every 1-dimensional complex (i.e. graph) has a perfect discrete Morse function, and in dimension 2 it is known that every closed, oriented surface has a $\mathbb{Z}$-perfect discrete Morse function [14], every closed surface has a $\mathbb{Z}_{2}$-perfect discrete Morse function $[\mathbf{1}]$, and every 2-dimensional subcomplex of a 2-manifold has a $\mathbb{Z}_{2}$-perfect discrete Morse function [12]. Spheres are characterized by the existence of a perfect discrete Morse function in the sense that every triangulated manifold with exactly two critical cells is a sphere, and for every sphere there exists a triangulation with exactly two critical cells [6]. Finding perfect discrete Morse functions is a difficult problem, as Joswig and Pfetsch [11] have shown it is NP-hard even on 2-dimensional complexes.

Before we finish this section, let us point out that along the process of (de)composing discrete Morse functions one may need to subdivide some of the cells in the complex. As in (cf. $[\mathbf{1 5}, \mathbf{6}]$ ), a bisection of a cell will be a subdivision of a single cell into two, as shown in Figure 2. 

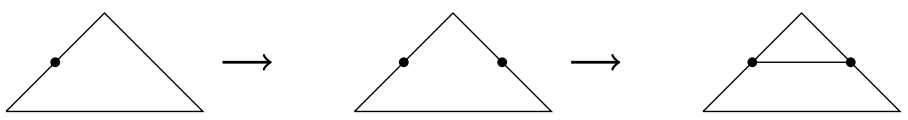

Figure 2: A sequence of bisections.

\section{Composing perfect discrete Morse functions on a connected sum}

In this section, we prove our first results. We show how to compose two perfect discrete Morse functions on triangulated manifolds. Our method is geometrically straightforward. The construction of the perfect discrete Morse function is similar to a method of Benedetti [5] to prove that perfect discrete Morse functions can be combined into a perfect discrete Morse function on the disjoint union, which he then used to show that a handlebody decomposition of a triangulated manifold gives a discrete Morse function on it with critical cells exactly corresponding to the handles in the decomposition, thus extending a similar result of Gallais [8] on 3-manifolds to the general case.

Let $M=M_{1} \# M_{2}$ be the connected sum of two closed, connected, oriented, triangulated $n$-dimensional manifolds, and $f_{1}$ and $f_{2}$ be perfect discrete Morse functions on $M_{1}$ and $M_{2}$ respectively. Since $M_{1}$ is closed and oriented, $H_{n}\left(M_{1}\right) \cong \mathbb{Z}$ so since $f_{1}$ is perfect, $m_{n}\left(f_{1}\right)=b_{n}\left(M_{1}\right)=1$, and also since $M_{2}$ is connected, $H_{0}\left(M_{2}\right) \cong \mathbb{Z}$ so since also $f_{2}$ is perfect, $m_{0}\left(f_{2}\right)=b_{0}\left(M_{2}\right)=1$. We form the connected sum $M=M_{1} \# M_{2}$ by removing an $n$-cell of $M_{2}$ with minimal vertex in its boundary and the critical $n$-cell of $M_{1}$. The next result can be thought of as a generalization of [3, Theorem 6 ] but the reader should note that the function that we are going to construct coincides with $f_{1}$ and $f_{2}$ except where the connected sum is formed.

Theorem 3.1. Let $M=M_{1} \# M_{2}$ be given as above. Then, there exists a polyhedral subdivision $\widetilde{M}$ of $M$ and a perfect discrete Morse function $f$ on $\widetilde{M}$ that agrees, up to a constant on each summand with $f_{1}$ and $f_{2}$, except on a neighborhood of the two removed cells.

Proof. In order to prove the theorem, we will show that the gradient vector fields $V_{1}$ and $V_{2}$ of the functions $f_{1}$ and $f_{2}$ respectively, induce a discrete vector field $V$ on the connected sum which restricts to $V_{1}$ and $V_{2}$ on the two summands, and which has the minimal possible number of critical cells and no loops. We may assume that the connected sum $M=M_{1} \# M_{2}$ is formed by removing a non-critical $n$-cell $\beta$ of $V_{2}$ with the minimal vertex $v$ in its boundary and the critical $n$-cell $\alpha$ of $V_{1}$.

On $M_{1} \backslash \alpha$, we attach a tube $L=\partial \alpha \times[0,1]$ with the natural product cell decomposition along $\partial \alpha \times\{0\}$ and extend the discrete vector field $\left.V_{1}\right|_{M_{1}-\alpha}$ to $\left(M_{1}-\alpha\right) \cup L$ so that each cell $\sigma$ in $\partial \alpha \times\{1\}$ is paired with the cell $\sigma \times(0,1)$ (see Figure 3 for $n=2$ ). Also note that if $\partial \alpha$ contains any critical 1-cells that belong to $M_{1}$, we may do bisections to push these cells in $M_{1}-\alpha$.

On $M_{2}$, we subdivide the smallest simplicial complex $J$ consisting of $\beta$ and all its faces in the following way. Let $H: J \times[0,1] \rightarrow J$ be a linear (deformation) retraction of $J$ onto the critical vertex $v$. Then, $J^{\prime}=H_{1 / 2}(J)$ represents a smaller copy of $J$ and $\beta^{\prime}=H_{1 / 2}(\beta)$. The closure $J^{\prime \prime}$ of the complement $J-J^{\prime}$ is a product of the link 

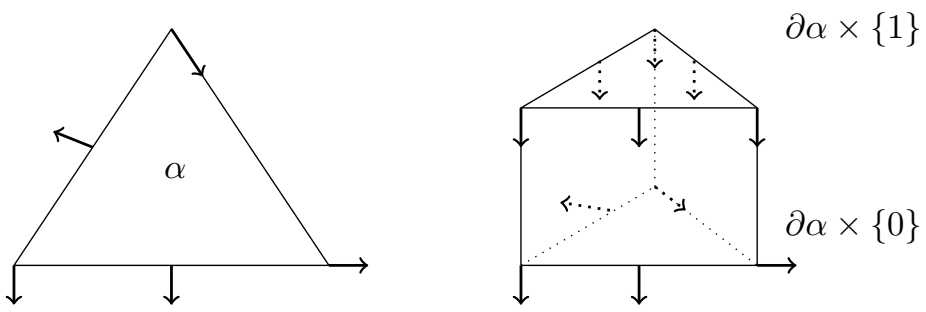

Figure 3: The discrete vector field on $\partial \alpha \times\{1\}$.

$\operatorname{Lk}_{J}(v)$ of $v$ in $J$ (that is, the face of $\beta$ opposite to $v$ with all its faces) with the interval $[0,1 / 2]$, and the simplices of $J^{\prime \prime}-J^{\prime}$ are in bijective correspondence to the simplices of $J-v$ (see Figure 4 for $n=2$ ).
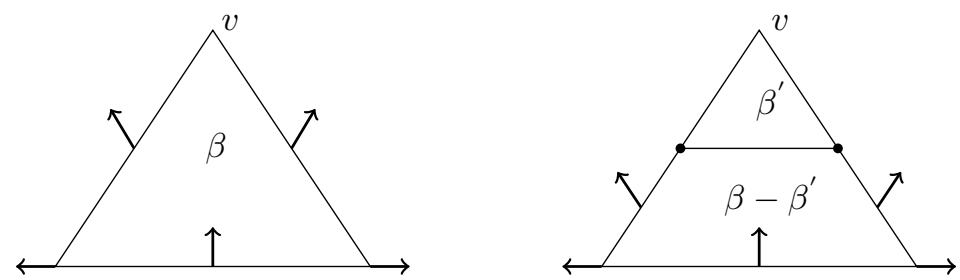

Figure 4: Obtaining $\beta^{\prime}$ with discrete vector field on it.

We extend the vector field $V_{2}$ on $M_{2}-J$ to $J^{\prime \prime}-J^{\prime}$ so that for any pair $(\sigma, \tau) \in V_{2}$ where $\sigma \in J$ and either $\tau \in J$ or $\tau \in\left(M_{2}-J\right)$, there is a corresponding pair $\left(\sigma^{\prime \prime}, \tau^{\prime \prime}\right)$ where $\sigma^{\prime \prime} \in J^{\prime \prime}$ and either $\tau^{\prime \prime} \in J^{\prime \prime}$ or $\tau^{\prime \prime} \in\left(M_{2}-J\right)$, and all simplices on $\partial \beta^{\prime}$ are unpaired. The connected $\operatorname{sum}(\widetilde{M})$ is formed by removing $\beta^{\prime}$ and identifying $\partial \beta^{\prime}$ with $\partial \alpha \times\{1\}$. Since each cell $\sigma \in \partial \beta^{\prime} \cong \partial \alpha \times\{1\}$ was unpaired in $V_{2}$, it can be without conflicts paired according to $V_{1}$ to the cell $\sigma \times[0,1]$ in $L$. The vector fields on $\left(M_{1}-\right.$ $\alpha) \cup L$ and $\left(M_{2}-\beta\right) \cup \beta^{\prime \prime}$, where $\beta^{\prime \prime}=\beta-\beta^{\prime}$, together form a vector field $V$ on $\widetilde{M}$ given by

$$
V(\gamma)= \begin{cases}V_{1}(\gamma) ; & \gamma \in M_{1}-\alpha, \\ \gamma \times(0,1) ; & \gamma \in \partial \alpha \times\{1\} \cong \partial \beta^{\prime}, \\ V_{2}(\sigma) ; & \gamma=\sigma^{\prime \prime} \in J^{\prime \prime}, \\ V_{2}(\gamma) ; & \gamma \in M_{2}-J .\end{cases}
$$

Since $V_{1}$ and $V_{2}$ do not contain loops and all the arrows on the boundary circle point in the same direction (towards $M_{1}$ ), there cannot be any closed $V$-paths, and we have a discrete gradient vector field on $\widetilde{M}$. Since the maximum $\alpha \in M_{1}$ has been removed and the minimum $v \in M_{2}$ has been paired, the numbers of critical cells in $V$ are

$$
\begin{aligned}
m_{0} & =1=b_{0}(M), \\
m_{i} & =m_{i}\left(f_{1}\right)+m_{i}\left(f_{2}\right)=b_{i}(M) \text { for } i=1, \ldots, n-1, \\
m_{n} & =1=b_{n}(M) .
\end{aligned}
$$


For example, assuming there is no pairing between the faces of the critical $n$-cell $\alpha$ of $f_{1}$ and the values of $f_{2}$ are greater than or equal to the values of $f_{1}$, a perfect discrete Morse function $f$ can be given by

$$
f(\gamma)= \begin{cases}f_{1}(\gamma) ; & \gamma \in M_{1}-\alpha \\ f_{1}(\tau)+C / 2 ; & \gamma=\tau \times(0,1), \gamma=\tau \times\{1\} \in L, \tau \in \partial \alpha \\ f_{2}(\gamma)+C ; & \gamma \in M_{2}-J \\ f_{2}(\tau)+C ; & \gamma=\tau^{\prime \prime} \in J^{\prime \prime}\end{cases}
$$

where $C$ is a big enough constant (bigger than $f_{1}(\alpha)+1$ ).

\section{Decomposing perfect discrete Morse functions on surfaces}

In this section we are going to prove the converse of Theorem 3.1 in the case of surfaces. That is, any perfect discrete Morse function $f$ on a closed triangulated surface $M$ that is a connected sum $M_{1} \# M_{2}$ restricts to perfect discrete Morse functions $f_{1}$ and $f_{2}$ on the two summands. Before we state our theorem let us first point out an observation for a discrete Morse function on a particular boundary curve of an oriented surface. Following [4] the cells in the boundary of a manifold with a discrete Morse function $f$ on it are called boundary critical cells if they are critical for $f$.

Lemma 4.1. Let $M$ be a closed, oriented surface of genus $g$ and $f$ be a discrete Morse function on $M$. Let $D$ be an open disk in $M$ which contains exactly one critical 0-cell or one critical 2-cell and let $C=\partial(M-D)$. Then the number of boundary critical vertices and edges of $\left.f\right|_{M-D}$ on $C$ must be equal.

Proof. The Euler characteristic of the closed genus $g$-surface $M$ is $\chi(M)=2-2 g$. Removing a disk decreases Euler characteristic by one. So, we have

$$
\begin{aligned}
\chi(M-D) & =2-2 g-1 \\
& =1-2 g .
\end{aligned}
$$

Assume that $D$ contains only one critical 0-cell of $f$ in $M$ (the argument is similar if $D$ contains only one critical 2-cell). Assume also that $f$ has $m_{0}$ critical 0 -cells, $m_{1}$ critical 1-cells and $m_{2}$ critical 2-cells. Let $n$ be the number of the boundary critical 0 -cells and $m$ be the number of the boundary critical 1-cells on $C$. Note that these 0 -cells and 1-cells are critical for $\left.f\right|_{M-D}$. Hence, $\left.f\right|_{M-D}$ has $\left(m_{0}+n\right)$ critical 0-cells, $\left(m_{1}+m\right)$ critical 1-cells and $m_{2}$ critical 2-cells. Then, by the Morse inequalities,

$$
\begin{aligned}
\chi(M) & =m_{0}-m_{1}+m_{2}=2-2 g, \text { and } \\
\chi(M-D) & =\left(m_{0}+n-1\right)-\left(m_{1}+m\right)+m_{2} \\
& =\left(m_{0}-m_{1}+m_{2}\right)+n-m-1 \\
& =(2-2 g)+n-m-1 \\
& =1-2 g+(n-m) .
\end{aligned}
$$

Equations (1) and (2) imply that $n=m$.

Indeed, $\left.f\right|_{C}$ is a discrete Morse function in Lemma 4.1 and

$$
\chi(C)=0=m_{0}\left(\left.f\right|_{C}\right)-m_{1}\left(\left.f\right|_{C}\right)
$$

implies that $m_{0}\left(\left.f\right|_{C}\right)=m_{1}\left(\left.f\right|_{C}\right)$ where $m_{0}\left(\left.f\right|_{C}\right), m_{1}\left(\left.f\right|_{C}\right)$ are the numbers of critical 
0-cells and critical 1-cells of $\left.f\right|_{C}$, respectively. However, our main goal in Lemma 4.1 is to show that if $C$ bounds a surface which is $M-D$, then the numbers of the boundary critical 0 -cells and 1-cells are equal.

In the proof of the main theorem of this section we will also need the following two well known results which we add for the sake of completeness of the paper.

Lemma 4.2. On any regular cell complex 1-paths can merge but cannot split. All 1-paths in the gradient field of a perfect discrete Morse function on a connected cell complex thus form a tree with root at the minimal vertex.

Proof. If a 1-path would split at some vertex, this would imply that the vertex is paired with two different edges which is clearly not possible. Every 1-path ends in a critical vertex. A connected cell complex has $b_{0}=1$ so a perfect discrete Morse function on it has one critical vertex which is the root of a tree formed by all 1paths.

Lemma 4.3. For $n \geqslant 2$, the $n$-paths in the gradient field of a discrete Morse function on a closed triangulated n-dimensional manifold can split but not merge. Moreover, for any $n \geqslant 1$, and every $n$-dimensional cell $\sigma$ of a triangulated $n$-manifold with a perfect discrete Morse function, with the exception of the unique critical $n$-cell, there exists a unique n-path beginning in the boundary of the critical $n$-cell and ending in $\sigma$.

Proof. In a closed triangulated $n$-manifold, every $(n-1)$-cell is the common face of exactly two $n$-cells. If two $n$-paths would merge at some $(n-1)$-cell $\tau$, then $\tau$ would be the common face of its $n$-dimensional pair as well as at least two other $n$-cells which is not possible. If $\sigma$ is an $n$-cell in an $n$-path, then its pair is an $(n-1)$-cell which has precisely one other $n$-coface. If this is not critical, it is the only possible previous $n$-cell in the path. Repeating this argument we eventually trace out a unique $n$-path starting in a critical $n$-cell.

Next we prove that a given perfect discrete Morse function on an oriented surface can be decomposed easily into two perfect discrete Morse functions if the separating curve is nice enough. Then in Theorem 4.6 we prove that one can always find a nice enough separating curve.

Let $M_{i}$ be a closed, oriented, triangulated genus $g_{i}$ surface and $D_{i} \subset M_{i}$ be an embedded disk for $i=1,2$. Also let $M=M_{1} \#_{C} M_{2}$ along the circle $C$. This circle separates $M$ into two parts, $M-\left(M_{1}-\operatorname{Int}\left(D_{1}\right)\right)$ and $M-\left(M_{2}-\operatorname{Int}\left(D_{2}\right)\right)$, which we will denote by abuse of notation as $M-M_{1}$ and $M-M_{2}$ i.e., $C \approx \partial\left(M-M_{1}\right) \approx$ $\partial\left(M-M_{2}\right)$ (how we form these regions and the naming will become clear throughout the proof of Theorem 4.6). Let $f$ be a perfect discrete Morse function on $M$ and $V$ be the gradient vector field induced by $f$ such that the restriction of $V$ to $M-M_{2}$ has one critical 0 -cell and $2 g_{1}$ critical 1-cells and the restriction of $V$ to $M-M_{1}$ has one critical 2-cell and $2 g_{2}$ critical 1-cells. Further, assume that there are no arrows on the vertices and edges of $C$ pointing into $M-M_{1}$ (later in Theorem 4.6 we are going to show that one can always find such a separating circle).

Theorem 4.4. Under the above conditions, we can extend $\left.V\right|_{M-M_{2}}$ to $M_{1}$ and $\left.V\right|_{M-M_{1}}$ to $M_{2}$ as perfect gradient vector fields induced by perfect discrete Morse functions which agree with $f$ on $M-M_{1}$ and $M-M_{2}$. 
Proof. Each cell on the boundary curve $C$ is paired either with a cell on $C$ or a cell in $M-M_{2}$. Assume that there are $k$ pairs of cells on $C$. Our aim is to obtain a perfect gradient vector field on $M_{2}$ after attaching a disk $D_{2}$ to $M-M_{1}$ in the following way. We triangulate the disk $D_{2}$ as a cone over $C$. That is, we choose an interior point, called $v$, in $D_{2}$ and connect $v$ with the vertices on $C$ via straight line segments. We pair each boundary critical cell of $M-M_{1}$ (each is paired in $M$ with a cell in $\left.M-M_{2}\right)$ with its coface in the cone. For each pair $(\sigma, \tau) \in V$ of a 0 -cell $\sigma$ and a 1-cell $\tau$ in $C$, the corresponding pair $\left(\sigma^{\prime}, \tau^{\prime}\right)$ inside the cone, where $\sigma^{\prime}$ and $\tau^{\prime}$ are cofaces of $\sigma$ and $\tau$ respectively, is formed. The vertex $v$ of the cone will be the critical vertex (see Figure 5 for $k=2$ ). The vector field $V$ has no non-trivial closed path in $M$, and hence $\left.V\right|_{M-M_{1}}$ has no non-trivial closed path. Additionally, we pair all boundary critical cells on $C$ with their cofaces in $D_{2}$, and hence, the extended vector field on $\left(M-M_{1}\right) \cup_{C} D_{2} \cong M_{2}$, called $V_{2}$, has no non-trivial closed path. Therefore, $V_{2}$ is the gradient vector field of a discrete Morse function [7, Theorem 3.5], say $f_{2}$, on $M_{2}$ with the following numbers of critical cells which tells that $V_{2}$ is a perfect gradient vector field:

$$
\begin{aligned}
& m_{0}=1=b_{0}\left(M_{2}\right), \\
& m_{1}=m_{1}\left(f_{2}\right)=b_{1}\left(M_{2}\right), \\
& m_{2}=1=b_{2}\left(M_{2}\right) .
\end{aligned}
$$

We can also obtain $f_{2}$ specifically by assigning values to the cells in $D_{2}$ so that they descend along the gradient paths, and the minimum is at the critical vertex $v$.

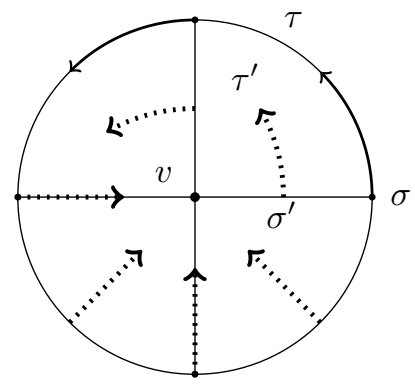

Figure 5: The discrete vector field on the disk with the critical 0-cell in the center.

Now, we construct a perfect gradient vector field on $M_{1}$ after attaching a disk $D_{1}$ to $M-M_{2}$. In this case, there are no boundary critical cells on $C$ since each cell on $C$ is paired either to a cell on $C$ or to a cell in $M-M_{2}$. Again, we triangulate the disk $D_{1}$ as a cone over $C$. Let $t_{i}$ be the triangle and $e_{i}, e_{i+1}$ the faces of $t_{i}$ for $i=1,2,3, \ldots, n$, which are ordered in counterclockwise direction in the interior of $D_{1}$. We pair $e_{i}$ 's with $t_{i}$ 's where $i=1,2,3, \ldots, n-1$ and pair $v$ with $e_{n}$. At the end, one unpaired triangle, $t_{n}$, will remain in $D_{1}$ since the number of edges and triangles in $D_{1}$ equal (see Figure 6 for $k=2$ and $n=4$ ).

We obtain a discrete vector field on $D_{1}$ with a critical 2-cell (see Figure 6). We do not get any non-trivial closed paths in $M_{1}$ after attaching $D_{1}$, since there is not any non-trivial closed path in $M-M_{2}$ and there is no $i$-path, $i=1,2$, which begins in boundary of a cell in $D_{1}$ and comes back to $D_{1}$. Hence, we obtain a gradient 


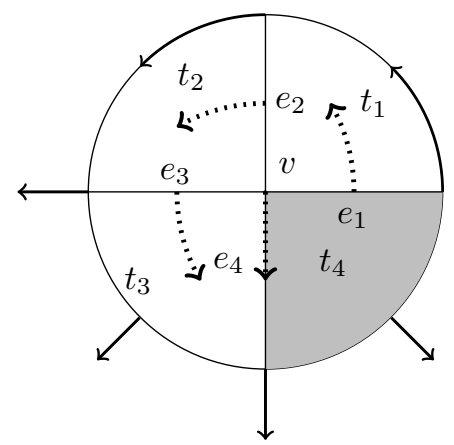

Figure 6: The discrete vector field on the disk with the critical 2-cell.

vector field $V_{1}$ induced by a discrete Morse function $f_{1}$ on $\left(M-M_{2}\right) \cup_{C} D_{1} \cong M_{1}$ [7, Theorem 3.5]. In addition, $V_{1}$ is the perfect gradient vector field of $f_{1}$ with the number of critical cells:

$$
\begin{aligned}
& m_{0}=1=b_{0}\left(M_{1}\right), \\
& m_{1}=m_{1}\left(f_{1}\right)=b_{1}\left(M_{1}\right), \\
& m_{2}=1=b_{2}\left(M_{1}\right) .
\end{aligned}
$$

Similarly, we can define $f_{1}$ specifically by assigning a value big enough to the critical triangle $t_{n}$ and descending values along the $V_{1}$-paths in $D_{1}$ keeping in mind that the values should all be bigger than the values on the circle $C$.

Remark 4.5. An alternative way to see that the discrete Morse function $\left.f\right|_{M-M_{2}}$ extends to $M_{1}$ as a perfect discrete Morse function is as follows: We triangulate the disc $D_{1}$ as a cone with a single interior vertex $v$. We choose one of the triangles in $D_{1}$, called $T$. Clearly, $\left(D_{1}-\operatorname{int}(T)\right) \searrow C$ (for the definition of collapse, see for example $[\mathbf{7}$, pp. 11-12]). Indeed, $\left(M-M_{2}\right) \cup_{C} D_{1} \cong M_{1}$ and $\left(M_{1}-\operatorname{int}(T)\right) \searrow\left(M-M_{2}\right)$. Hence, $\left.f\right|_{M-M_{2}}$ can be extended to $M_{1}-T$ as a discrete Morse function without obtaining any new critical cells by following the inverse of the collapse steps of $\left(D_{1}-\operatorname{int}(T)\right) \searrow$ $C\left[\mathbf{6}\right.$, Lemma 4.3]. Let $g$ be the extension of $\left.f\right|_{M-M_{2}}$ to $\left(M_{1}-T\right)$. Then, we define a discrete Morse function on $M_{1}$ as in the following way:

$$
g^{\prime}(\sigma)= \begin{cases}g(\sigma) ; & \sigma \in M_{1}-T, \\ \max \{g(\partial \sigma)\}+c ; & \sigma=T .\end{cases}
$$

Therefore, $g^{\prime}$ is a perfect discrete Morse function with a unique critical 2-cell $T$.

Now, let $M=M_{1} \# M_{2}$ be the connected sum of two closed oriented triangulated surfaces of genus $g_{1}$ and $g_{2}$ respectively. Let $f$ be a perfect discrete Morse function on $M$ such that the critical 2-cell of $f$ is in $M-M_{1}$ and the critical 0-cell of $f$ is in $M-M_{2}$. In addition, assume that the critical cells of $f$ are separated in the sense that the star of a critical cell contains no other critical cells. This can always be achieved after a suitable subdivision of $M$. Let $V$ be the gradient vector field induced by $f$. In the following theorem, we look for a suitable boundary curve $C$, such as in Theorem 4.4, so that one can decompose $f$ accordingly, and thus one can decompose $M$ along the boundary curve (separating circle) $C$. 
The first thing one needs to do is to decide which critical cells belong to the same component. Note that $M$ is a genus $g$ surface for some $g$. We may take $\left\{\alpha_{1}, \beta_{1}, \ldots\right.$, $\left.\alpha_{g}, \beta_{g}\right\}$ as a basis for $H_{1}(M)$, where $\alpha_{i}$ 's and $\beta_{i}$ 's are given by the critical 1 -cells of $f$. These homology classes are obtained by our perfect discrete Morse function following the 1-paths emanating from the critical 1-cells. Note that in any basis for the first homology group of a closed, connected, orientable surface of genus $g$, homology generators come as pairs. That is for any $\alpha$ in a basis for $H_{1}(M)$, there should be a class $\beta$ such that the number of transverse intersections is odd between any representatives of $\alpha$ and $\beta$. This follows from the fact that $H^{2}(M) \cong \mathbb{Z}$ and a generator is given by the cup product of two 1-dimensional cohomology generators, say $a$ and $b$, such that $(a \cup b)[M]=1$. In other words, $a([M] \cap b)=1$. In terms of homology, this can be explained as the cohomology classes $a$ and $b$ have Poincare duals intersecting transversally at an odd number of points (i.e. these classes should have an odd geometric intersection and their algebraic intersection should be 1). One cannot count these intersection numbers by considering the 1-paths from the critical cells. Because these paths do not intersect transversally but instead they may merge together. To get the correct pairing we are going to work with the dual homology generators obtained from the 2-paths from the critical 2-cell to the critical 1-cells.

Theorem 4.6. Let $M=M_{1} \# M_{2}$ be as above. Then we can find a circle $C$ on $M$ such that $M=M_{1} \#_{C} M_{2}$ and the cells on $C$ are paired with either the cells on $C$ or the cells in $M-M_{2}$ or both.

Proof. To find the separating circle $C$ as mentioned above, we follow the flow induced by $f$ (the direction along which $f$ is non-increasing) from the critical 2-cell to the critical 1-cells which belong to $M-M_{1}$. We consider the 2-paths that begin from a face of the critical 2-cell and end at these critical 1-cells. Each critical 1-cell has precisely two 2-cofaces, and according to Lemma 4.3 each one of these is the end of precisely one 2-path that begins in a face of the critical 2-cell. If the critical 2-cell is removed, we may collapse along these paths, starting at a free edge in the boundary of the critical 2-cell. These collapses produce tunnels with cells in the boundary that are paired either to cells in the boundary or to cells in the remaining part of the manifold. The critical 2-cell together with these tunnels and the critical 1-cells at their ends will represent the core of $M-M_{1}$. The boundary of this region is a curve $C^{\prime}$. If this curve is a circle, we have achieved our goal since this circle separates the region that has been removed and represents $M-M_{1}$ and the arrows along the curve point either along the curve or into the remaining part $M-M_{2}$.

Note that $C^{\prime}$ might not be a circle due to the fact that the triangulation on $M$ might be too coarse to allow the construction of such a circle. The problem is that two different tunnels, that is, two paths from the critical 2-cell to critical 1-cells in $M-M_{1}$ come too close together and meet in a vertex or are separated only by an edge, or more generally, by a 1-path. As a result of this, the curve $C^{\prime}$ is a union of wedges of several circles possibly with additional paths in the interior of $M-M_{1}$ that connecting the wedges. In order to obtain a separating curve that is a circle, we have to do some subdivisions. For instance, in Example 4.9, the resulting boundary of several 2-paths meet at the vertex 3 , so the curve $C^{\prime}$ is a disjoint union a wedge of circles meeting at this vertex and a circle, and the 1-path $a$ represents an additional 
edge (arc) joining these disjoint boundary components, since two 2-paths meet in this edge.

Case 1: Assume first that the curve $C^{\prime}$ is a wedge of $n$ circles at a single vertex $v$. First, we bisect all the 1-cells in the open star of $v$ in the tunnel (see Figure 7 for $n=2$ ). We always pair all the new vertices with their cofaces in the new star of
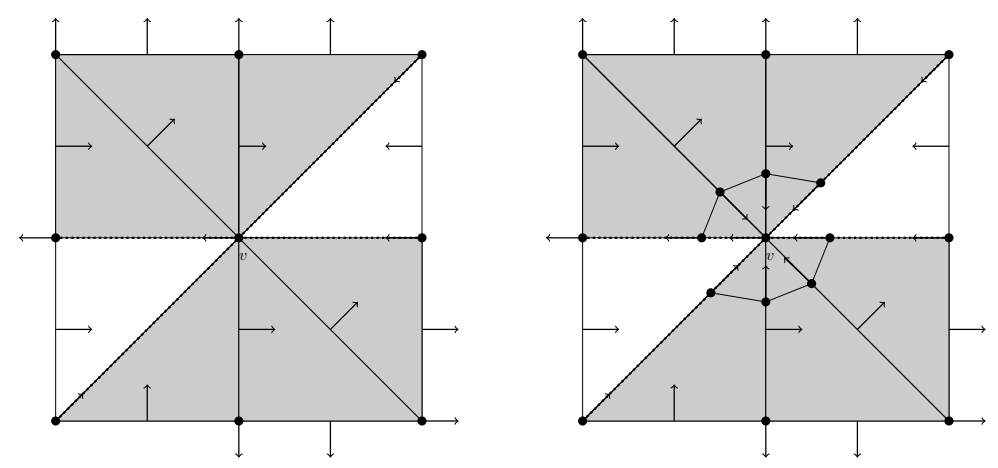

Figure 7: From left to right: The 2-paths meeting at the vertex $\nu$ and the bisections done in the open star of $\nu$.

$v$ obtained after bisections if $v$ is not paired with a 1-cell on the boundary of the tunnel. But, if $v$ is paired with a 1-cell $\alpha$ on the boundary, we pair the new vertex bisecting $\alpha$ with its unpaired coface, and we pair the remaining unpaired vertices with their cofaces containing $v$. Next, we bisect all the 2-cells in the open star of $v$ in the tunnel. Then we pair all the unpaired 1-cells with their cofaces in the new star of $v$, which is obtained after the last bisections in $M$, by using the method given in $[\mathbf{6}$, Theorem 12.1] (e.g. see Figure 8). Hence we extend the vector field to the subdivided

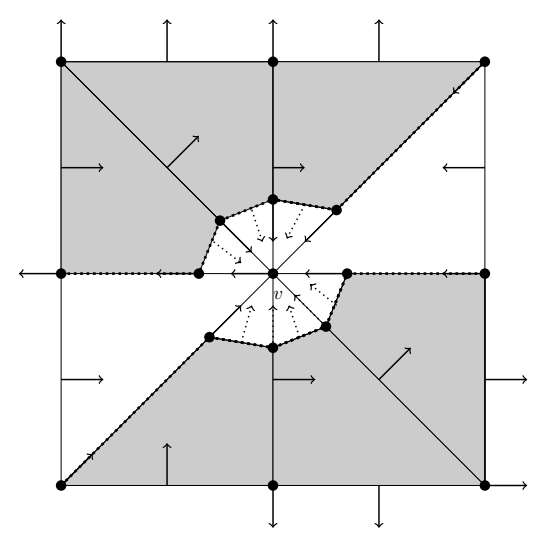

Figure 8: The separation of the 2-paths and extension of the vector field to the bisected cells.

cells without creating any non-trivial closed paths.

In this case, after the above process is completed, we collapse along the 2-paths, 
which are in the locally subdivided $M$ above, up to the critical 1-cells that belong to $M-M_{1}$. Since we separate the 2-paths meeting in the vertex $v$, the resulting boundary is a circle which is our boundary curve $C^{\prime}$ (see Figure 8 ).

In Figures 7 and 8, the gray regions represent a part of the interior of the tunnel while the white ones are a part of the outside of the tunnel, and the dotted edges represent the boundary curves.

Case 2: The curve $C^{\prime}$ might be a union of wedges of several circles with additional arcs connecting them. Note that the resulting boundary of $C^{\prime}$ is disconnected, i.e., it is the disjoint union of curves such that these curves are connected via interior additional arcs (1-paths) in the tunnel. Firstly, we will obtain a connected boundary curve via connecting disjoint boundary components by pushing the interior arcs of the tunnel outside after doing some subdivisions as in the following explanation.

Let $a$ be the additional arc (1-path) connecting disjoint boundary components of $C^{\prime}$, and $S$ be the star of $a$ in the tunnel (see Figure 9). We first bisect all the 1-cells
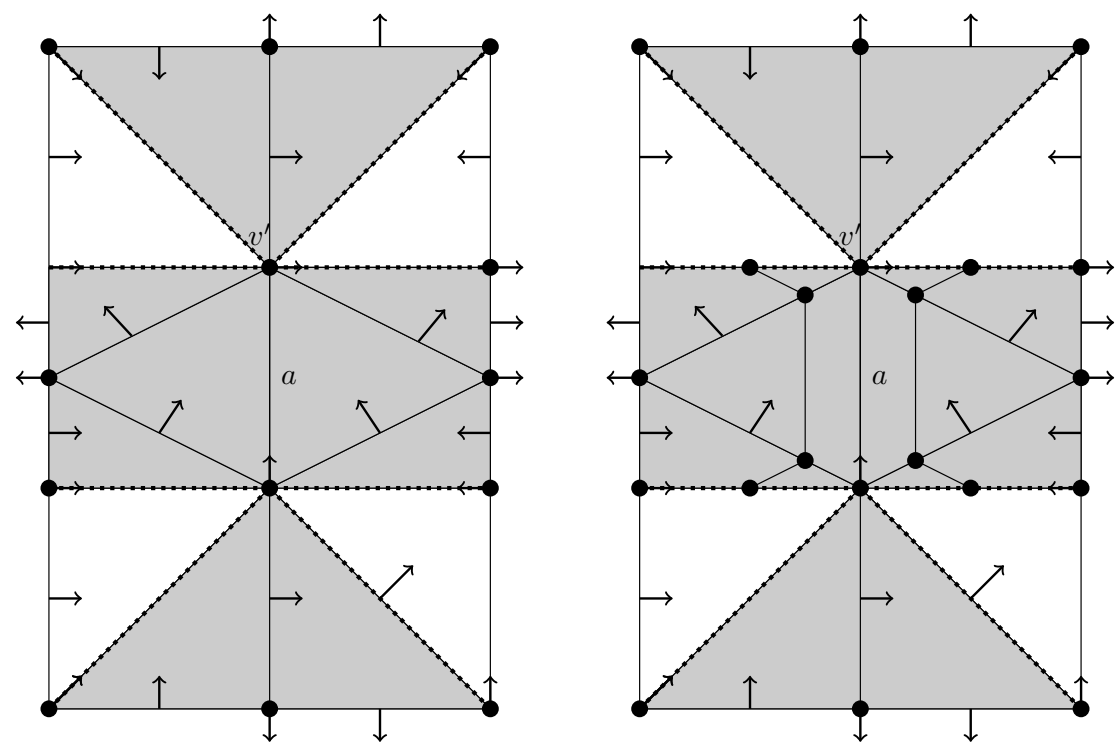

Figure 9: From left to right: The 2-paths separated by the 1-path $a$ and the bisections done in the star of $a$.

in $S-a$ which have non-trivial intersection with $a$. Then we pair all the new vertices with their cofaces in the new star of $a$, which obtained after the bisections on the 1-cells, if the terminal point $v^{\prime}$ of $a$ on $C^{\prime}$ is not paired with a 1-cell on $C^{\prime}$. But, if $v^{\prime}$ is paired with a 1 -cell $\beta$ on $C^{\prime}$, then we pair the new vertex bisecting $\beta$ with its unpaired coface, and we pair the remaining vertices with their cofaces in the star of $v^{\prime}$ (see Figure 9). Next, we bisect all the 2-cells in $S$ by connecting the new vertices bisecting the 1-cells above. We pair all the unpaired 1-cells, which is obtained after the last bisections in $M$, with their cofaces in the new star of $v^{\prime}$. For all the bisections and parings here, we use the method given in [6, Theorem 12.1], and thus we extend the vector field to the subdivided cells without creating any cycles. 
After eliminating all such additional arcs (1-paths) by using the process above, and collapsing along the 2-paths, which are in the lastly subdivided $M$ in the above process, up to the critical 1-cells in $M-M_{1}$, the resulting boundary of the tunnel is connected since we separate the paths which are separated by a 1-path $a$. It is either a circle, a wedges of circles or several wedges of circles. If it is a circle, then we have succeed our aim. But, if it contains a union of wedges of circles, then we use the method given in Case 1 to get rid of all wedge points and obtain a single circle as boundary curve $C^{\prime}$.

Remark 4.7. Note that in Theorem 4.6, we need to do subdivisions to obtain the boundary as a manifold. The boundary of the 2-manifolds we get should be a circle after the subdivisions (the boundary will be a closed 1-dimensional manifold).

The following is an immediate corollary of Theorems 4.4 and 4.6.

Corollary 4.8. Let $M=M_{1} \# M_{2}$ be the connected sum of two closed oriented triangulated surfaces of genus $g_{1}$ and $g_{2}$ respectively and $f$ be a perfect discrete Morse function defined on $M$. Then we can extend $f_{\left.\right|_{M-M_{2}}}$ to $M_{1}$ and $f_{\left.\right|_{M-M_{1}}}$ to $M_{2}$ as perfect discrete Morse functions.

To clear up the process described in Theorem 4.6, we work it out in the following example.

Example 4.9. Let $M$ be the triangulated genus 2 orientable surface with a perfect discrete Morse function which induces the gradient vector field depicted in Figure 10.

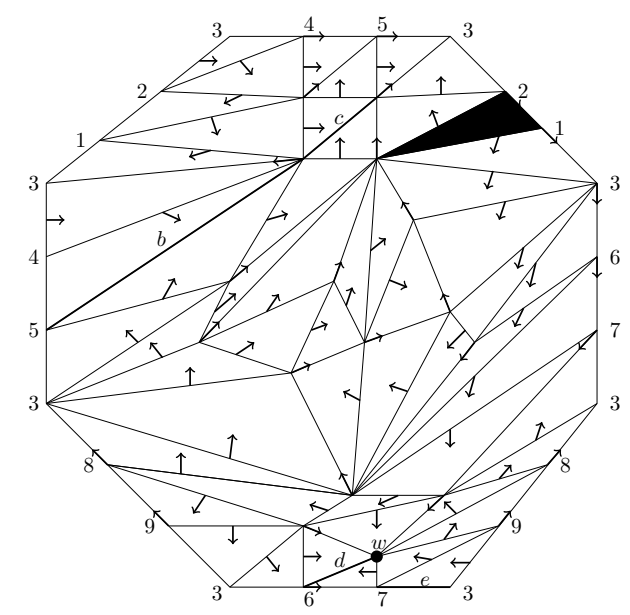

Figure 10: A gradient vector field on genus 2 orientable surface.

The black triangle is the critical 2-cell and the vertex $w$ is the critical 0-cell. The edges $b, c, d$ and $e$ are the critical 1-cells. By Theorem 4.6, the critical 1-cells $b$ and $c$ belong to $M-M_{1}, d$ and $e$ belong to $M-M_{2}$. Because of our construction (see also the proof of Theorem 4.4) we do not want to see any critical cells on the resulting boundary. If a 2-path from the boundary of the critical 2-cell to a critical 1-cell 
passes through other critical cells, then we need to perturb the vector field by doing subdivision (bisection or barycentric subdivision) in the star of the critical cell.

The critical 1-cells $b$ and $c$ have a common vertex. Thus we separate them by using bisections (see Figure 11).

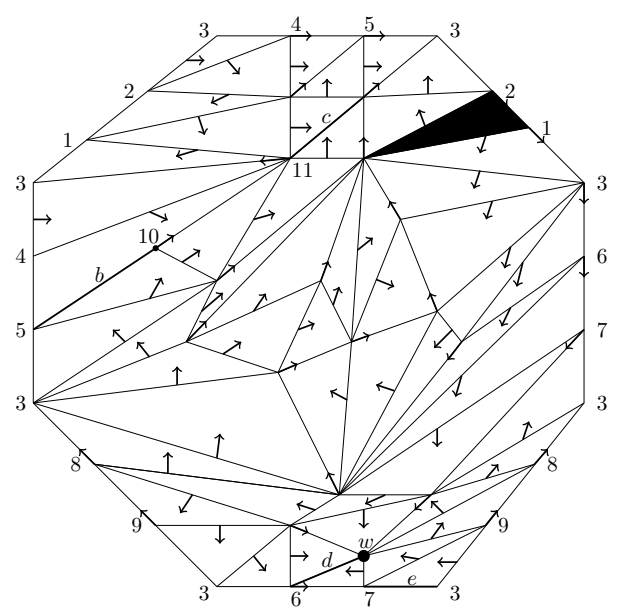

Figure 11: Separation of the critical edges.

The gray triangles show the 2-paths that end at the critical edges in Figure 12. First, we delete the interior of the critical 2-cell and then begin to collapse free edges

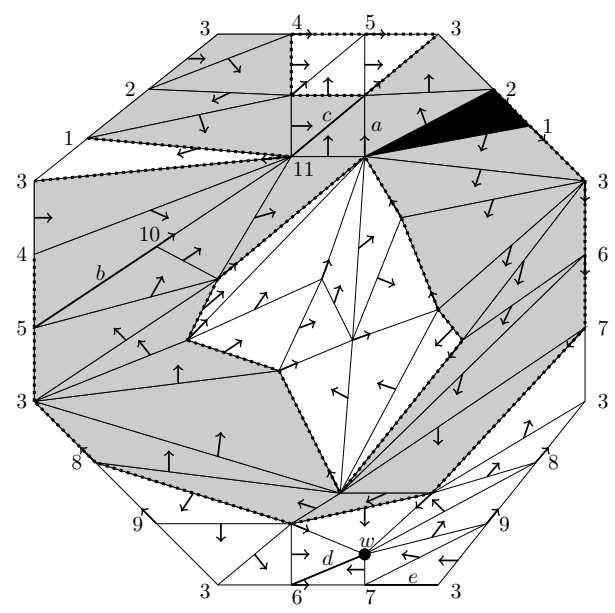

Figure 12: The 2-paths end at the critical edges.

and triangles that paired together in these 2-paths up to the critical 1-cells $b$ and $c$. The gray colored region is $M-M_{1}$, while the white region is $M-M_{2}$. The dotted curve is the boundary curve and the edge $a$ is the connecting 1-path (additional edge) between two disjoint boundary components. Note that the connecting edge $a$ does not belong to the boundary curve, that is, $a \in \operatorname{Int}\left(M-M_{1}\right)$. Note also that the edges 
$2-1,10-11$ are not boundary components. They are interior edges of $M-M_{1}$. In addition, the critical 1-cells $b$ and $c$ are the interior edges of $M-M_{1}$. Therefore, the boundary curve is a disjoint union of a circle and a wedge of three circle at the point 3 as on the figure left in Figure 13. These disjoint curves are connected in $M-M_{1}$ via the edge $a$.

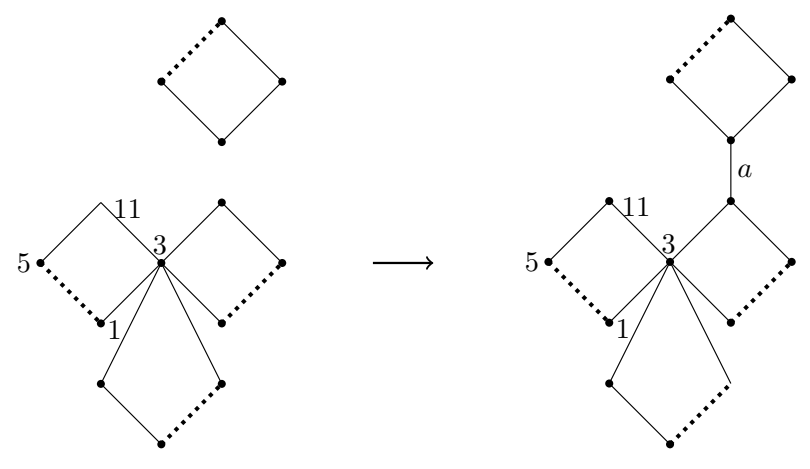

Figure 13: The boundary curve.
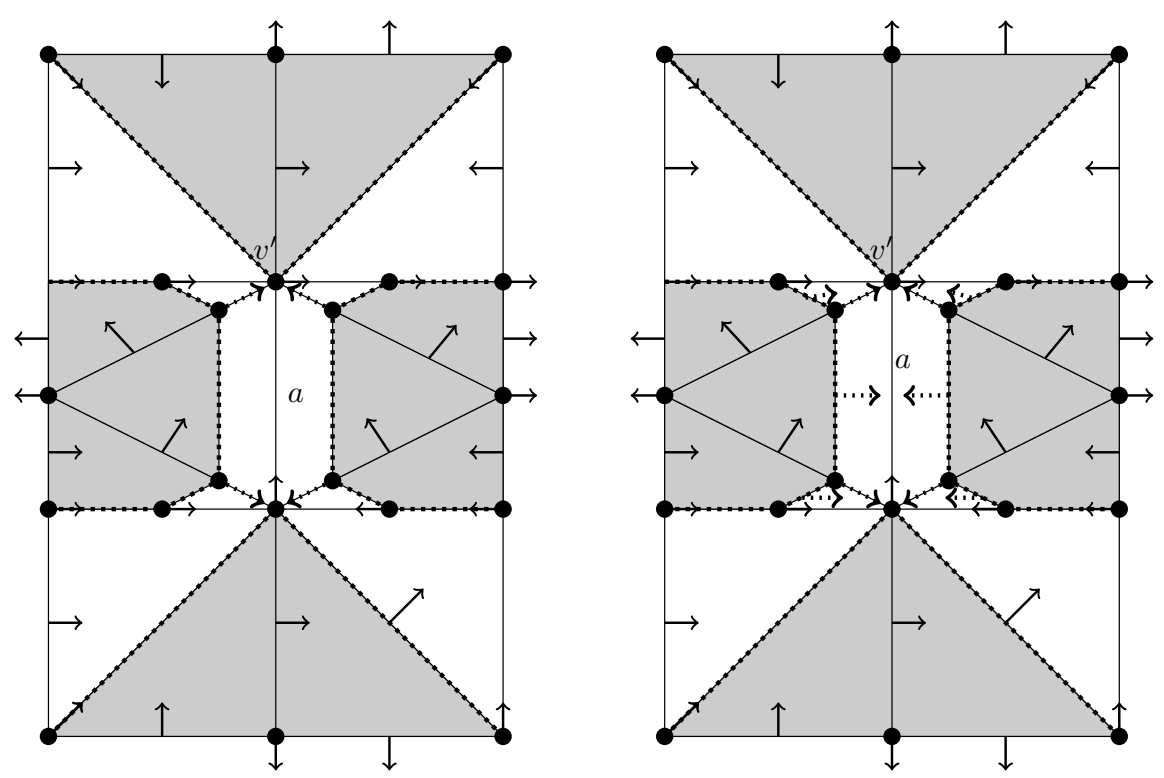

Figure 14: Extension of the vector field to the bisected cells in the star of $a$.

In Figure 13, the figure on the left is the boundary curve obtained after collapsing free edges and triangles up to critical edges, and the figure on the right denote the connecting edge between disjoint boundary components of the boundary curve. Then, we use the methods given in Figures 9, 14 (see Figure 15) to get rid of the connecting edge and connect the disjoint boundary components. 


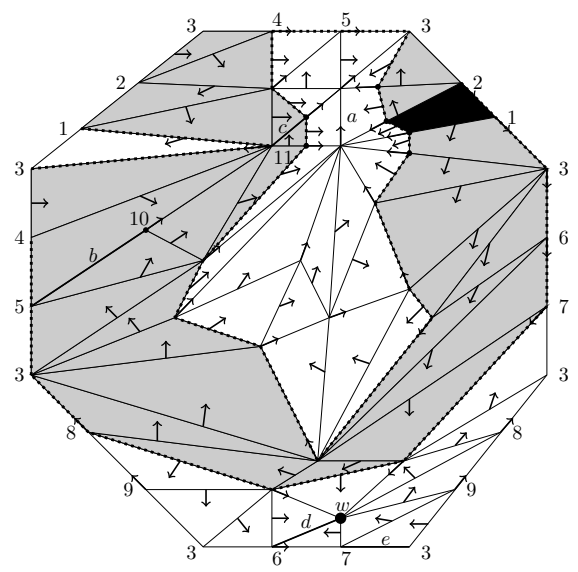

Figure 15: A separation of the 2-paths meeting along the 1-path $a$.

Now, the dotted curve is a wedge of three circles at the point 3 . We get rid of this wedge point by using the method in Figures 7 and 8 (see Figure 16). We pair all the

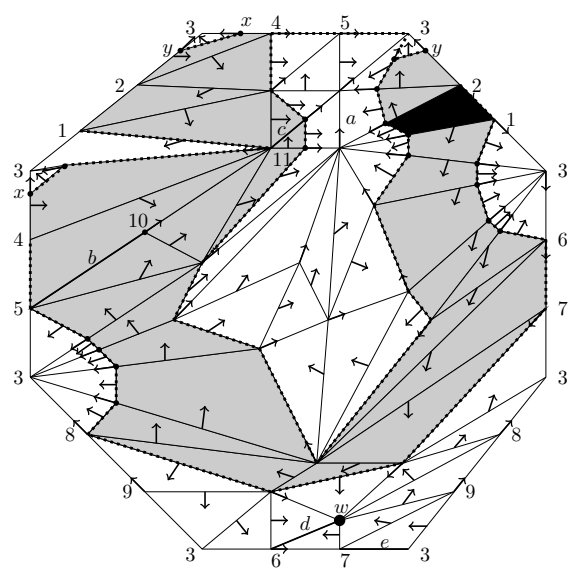

Figure 16: A separation of the 2-paths meeting in the vertex numbered 3.

cells on the new boundary components as we mention in the proof of Theorem 4.6. At the end, the boundary curve is a separating circle on $M$ as we want.

\section{References}

[1] R. Ayala, D. Fernández-Ternero and J.A. Vilches, Perfect discrete Morse functions on 2-complexes, Pattern Recogn. Lett. 33(11) (2012), 1495-1500.

[2] R. Ayala, L.M. Fernández and J.A. Vilches, Characterizing equivalent discrete Morse functions, Bull. Braz. Math. Soc. (N.S.) 40(2) (2009), 225-235. 
[3] R. Ayala, D. Fernández-Ternero and J.A. Vilches, Perfect discrete Morse functions on triangulated 3-manifolds, Computational Topology in Image Context. Lecture Notes in Computer Sci., Springer, Heidelberg 7309 (2012), 11-19.

[4] B. Benedetti, Discrete Morse theory for manifolds with boundary, Trans. Amer. Math. Soc. 364(12) (2012), 6631-6670.

[5] B. Benedetti, Smoothing discrete Morse theory, Ann. Sc. Norm. Super. Pisa Cl. Sci. (5) 16(2) (2016), 335-368.

[6] R. Forman, Morse theory for cell complexes, Adv. Math. 134(1) (1998), 90-145.

[7] R. Forman, A user's guide to discrete Morse theory, Sem. Lothar. Combin. 48 (2002), Art. B48c, 35 pp.

[8] E. Gallais, Combinatorial realizations of the Thom-Smale complexes via discrete Morse Theory, Ann. Sc. Norm. Super. Pisa Cl. Sci. (5) 9(2) 2010, 229252.

[9] S. Harker, K. Mischaikow, M. Mrozek and V. Nanda, Discrete Morse theoretic algorithms for computing homology of complexes and maps, Found. Comput. Math. 14(1) (2014), 151-184.

[10] P. Hersh, On optimizing discrete Morse functions, Adv. in Appl. Math. 35(3) (2005), 294-322.

[11] M. Joswig and M.E. Pfetsch, Computing optimal Morse matchings, SIAM J. Discrete Math. 20(1) (2006), 11-25.

[12] H. King, K. Knudson and N. Mramor, Generating discrete Morse functions from point data, Exp. Math. 14(4) (2005), 435-444.

[13] N.M. Kosta, M. Pamuk and H. Varl,, Decomposing perfect discrete Morse functions on connected sum of 3-manifolds, in preparation.

[14] T. Lewiner, H. Lopes and G. Tavares, Optimal discrete Morse functions for 2-manifolds, Comput. Geom. 26(3) (2003), 221-233.

[15] J.R. Stallings, Lectures on Polyhedral Topology, Notes by G. Ananda Swarup. Tata Inst. Fundam. Res. Lect. Math., No.43, Tata Institute of Fundamental Research, Bombay, 1967.

HanIfe Varlı hisal@metu.edu.tr

Mathematics Department, Middle East Technical University, Ankara, 06531, Turkey

Mehmetcik Pamuk mpamuk@metu.edu.tr

Mathematics Department, Middle East Technical University, Ankara, 06531, Turkey

Neža Mramor Kosta neza.mramor@fri.uni-lj.si

Faculty of Computer and Information Science and Institute of Mathematics, Physics and Mechanics, University of Ljubljana, Jadranska 19, Ljubljana, Slovenia 